\title{
THEORETICAL STUDY OF THE EFFECT OF MAGNETO-HYDRODYNAMICS AND COUPLE STRESS ON SQUEEZE FILM LUBRICATION OF ROUGH TRIANGULAR PLATES
}

\section{BIRADAR KASHINATH, HANUMAGOWDA B. N., SHARANAGOUDA MALIPATIL \& SHILPA DESAI}

Department of Mathematics, Govt. Degree College Kalaburagi, Karnataka, India

Department of Mathematics, School of Applied Sciences, REVA University, Karnataka, India

Department of Mathematics, Poojya Doddappa Appa Engineering College, Kalaburagi, Karnataka, India

Department of Mathematics, Faculty of Engineering and Technology, SharnbasvaUniversity, Kalaburagi, Karnataka, India

\begin{abstract}
In this paper, a theoretical analysis of the effects of magnetic field and couple stress on the squeeze film lubrication characteristics between rough triangular plates is presented. A generalized form of surface roughness is considered, and the modified average Reynolds equation is derived based onstochastic random variable with non-zero mean, variance and skewness with Magnetic field and couple stress fluid. Closed form solution is derived for squeeze film pressure, load carrying capacity and squeeze film time. Our result shows that there is a significant increase for pressure, load carrying capacity and squeeze film time due to magnetic field and couple stress lubricant, while these non-dimensional parameters decreases due to the standard deviation. It is noted that negatively skewed roughness increases the pressure, load carrying and squeezing time.
\end{abstract}

KEYWORDS: Couple Stress, Magneto Hydrodynamics, Triangular Plates

Received: Jun 08, 2020; Accepted: Jun 28, 2020; Published: Aug 20, 2020; Paper Id.: IJMPERDJUN2020833

\section{INTRODUCTION}

In engineering application, the magnetohydrodynamics (MHD) is the physical mathematical work that relates to the electrically conducting fluids. Such fluids are liquid metals,electrolytes, and salt water etc. In the recent trends various problems are solved on the motion of an electrically conducting liquid lubricant in MHD theory of lubrications. Many researchers analyzed theeffect of study of magnetic field, Huges and Elco[1] present MHD lubrication flow between parallel rotating disks.They analyzed that effect of MHD increase the load carrying capacity. Kuzma [2] presented the MHD journal bearing and reported that the load carrying capacity is increased by the applications of a magnetic field. The MHD squeeze film was studied by Kuzma, Makiand Donelly[3].Davies[4] have analyzed on the generation pressure between the rough fluid lubricated moving deformable surface and observed that the magnetic fluid lubricant improves the stability of bearing ,also found the increases of load carrying capacity due to the effect of applied magnetic fluid. Kumza[5] presented the MHD journal bearing. They observed that the load carrying capacity is increased by the application of magnetic field.To understand different physical problems, the concept ofcouple stress fluids is particularly important. The Newtonian classical theory cannot explain rheological properties of lubricants mixed with different additives; several theories are recommended to model the flow rheology, but Stoke's [6] theory is the simplest one that describes couple's stress concepts.Bujurke and Jayaraman [7]studiedinfluence of couple stresses in squeeze films. Ramanaiah [8] 
analyzed the Squeeze films between finite plates lubricated by couple stress fluid. Lin[9] examined squeeze film between a sphere and flat plate using couple stress fluid model.MHD squeeze film characteristics for finite rectangular plateswas studied by Lin [10].

Squeeze film lubricantion between circular stepped plates with couple stress fluids is examined by Naduvinamani and Siddangouda [11] and concluded that the couple stress parameter increases in the film pressure, load carrying capacity and response time as compared to classical Newtonian lubricant.The combine effect of MHD and couple stressfluid is studied by Naduvinamani et al [12-13] for different bearings and concluded that the effect of magnetic field and couple stress fluid is to enhance the squeeze film characteristics.

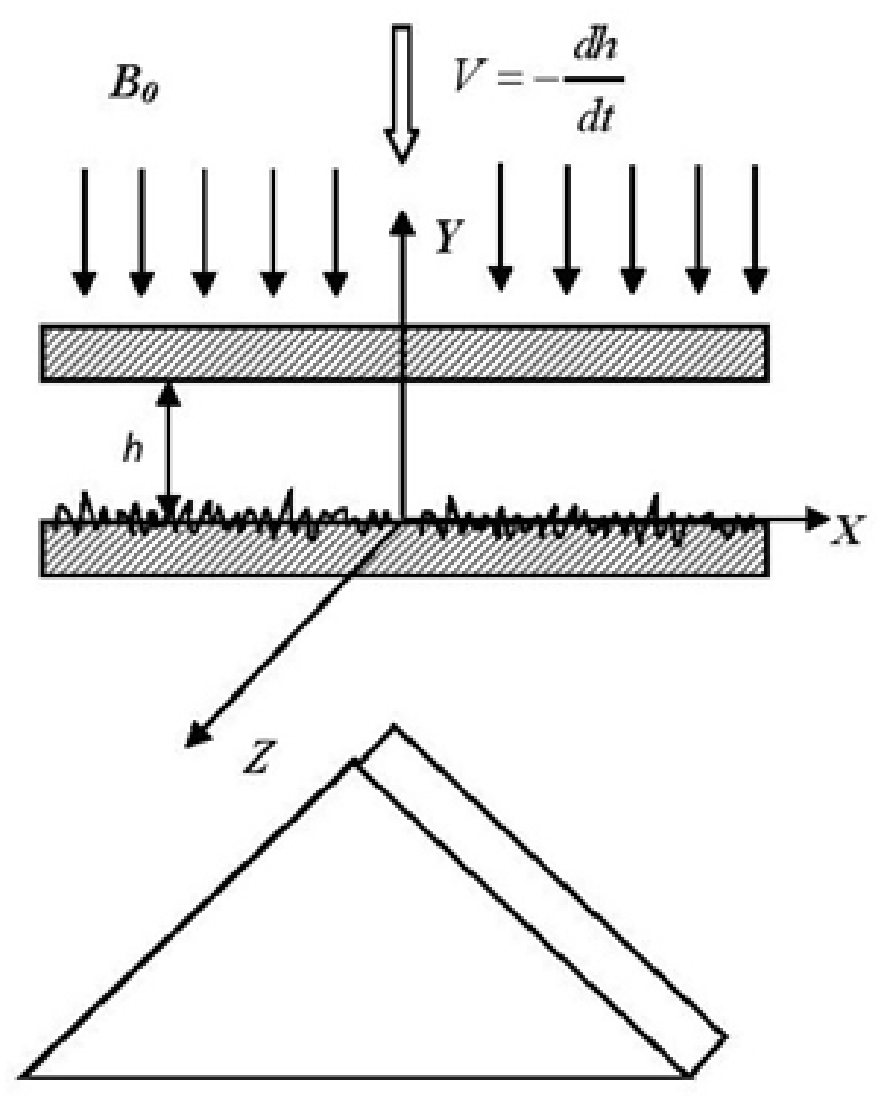

Figure 1: Schematic Diagram of the Rough Triangular Plate.

Once the gap among two contacting surfaces is reduced, the roughness effect become more significant. The smooth bearing surface would not be valid for predicting the performance and life of the bearings in most applications. Thus, surface roughness has been studied with much concern currently, since all bearing surfaces are rough to some extent. To enhance the performance of hydrodynamic lubrication in distinctive bearings, the study of surface roughness is important. Turaga [14] presented the hydrodynamic journal bearing with rough surface. The combined effect of surface roughness and MHD with couple stress fluid is investigated by many authors [15-24] and from their results it was concluded that the squeeze film attributes is significant. So far in the literature survey, no theoretical investigation is done on analysis of MHD- Couple stress Squeeze film lubrication of rough triangular plates. Hence in this paper, work has been carried out to study the effect of magnetic field with couple stress fluid on the rough triangular plates. 


\section{MATHEMATICAL FORMULATION}

The geometry and the configuration of the bearing system is as shown fig. 1. The lower plate is assumed to be fixed while the upper plate moves normally towards the lower plate with a uniform velocity $d h / d t$. The lubricant in the film region is assumed the couple stress fluid. It is assumed that the fluid film is thin, body forces and fluid inertia are negligible except for the Lorentz force and induced magnetic field is neglected compared to the applied magnetic field. Under these assumptions, the MHD momentum equations and the continuity equation with couple stress fluids are

$$
\begin{aligned}
& \frac{\partial^{2} u}{\partial y^{2}}-\frac{\eta}{\mu} \frac{\partial^{4} u}{\partial y^{4}}-\frac{\mu M_{0}^{2}}{\mu h_{0}^{2}} u=\frac{1}{\mu} \frac{\partial p}{\partial x} \\
& \frac{\partial^{2} v}{\partial y^{2}}-\frac{\eta}{\mu} \frac{\partial^{4} v}{\partial y^{4}}-\frac{\mu M_{0}^{2}}{\mu h_{0}^{2}} v=\frac{1}{\mu} \frac{\partial p}{\partial z} \\
& \frac{\partial p}{\partial y}=0 \\
& \frac{\partial u}{\partial x}+\frac{\partial v}{\partial z}+\frac{\partial w}{\partial y}=0
\end{aligned}
$$

Where $\mathrm{u}, v$ and $w$ are the fluid velocity components along $x, y$ and $z$ directions respectively in the film region, $p$ is the pressure in the film region, $\eta$ is the material constant characterizing the couple stress, $\mu$ is the material constant of dimension of viscosity.

The relevant boundary conditions are

At the upper surface $y=h$

$$
\begin{aligned}
& u=v=0, \frac{\partial^{2} u}{\partial y^{2}}=\frac{\partial^{2} v}{\partial y^{2}}=0 \\
& w=-\frac{d h}{d t}
\end{aligned}
$$

At the lower surface $y=0$

$$
\begin{aligned}
& u=v=0, \frac{\partial^{2} u}{\partial y^{2}}=\frac{\partial^{2} v}{\partial y^{2}}=0 \\
& w=0
\end{aligned}
$$

The solution of the equations (1) and (2) subject to the boundary conditions (5a) and (6a) is obtained in the form

$$
u=-\frac{h_{0}^{2}}{\mu M_{0}^{2}} \frac{\partial p}{\partial x}\left\{\frac{1}{\left(A^{2}-B^{2}\right)}\left(\frac{B^{2} \operatorname{Cosh} \frac{A(2 y-h)}{2 l}}{\operatorname{Cosh} \frac{A h}{2 l}}-\frac{A^{2} \operatorname{Cosh} \frac{B(2 y-h)}{2 l}}{\operatorname{Cosh} \frac{B h}{2 l}}\right)+1\right\}
$$

Similarly, 


$$
v=-\frac{h_{0}^{2}}{\mu M_{0}^{2}} \frac{\partial p}{\partial z}\left\{\frac{1}{\left(A^{2}-B^{2}\right)}\left(\frac{B^{2} \operatorname{Cosh} \frac{A(2 y-h)}{2 l}}{\operatorname{Cosh} \frac{A h}{2 l}}-\frac{A^{2} \operatorname{Cosh} \frac{B(2 y-h)}{2 l}}{\operatorname{Cosh} \frac{B h}{2 l}}\right)+1\right\}
$$

where, $M_{0}=B_{0} h_{0}(\sigma / \mu)^{1 / 2}$ is the Hartmann number $A=\left\{\frac{1+\left(1-4 M_{0}{ }^{2} l^{2} / h_{0}^{2}\right)^{\frac{1}{2}}}{2}\right\}^{\frac{1}{2}}, B=\left\{\frac{1-\left(1-4 M_{0}{ }^{2} l^{2} / h_{0}^{2}\right)^{\frac{1}{2}}}{2}\right\}^{\frac{1}{2}}$

Substituting (8) \& (9) in the continuity equation (5) and integrating across the film thickness $h$ and use of the boundary conditions (6) and (7) gives the Reynolds equation in the form

$$
\frac{\partial^{2} p}{\partial x^{2}}+\frac{\partial^{2} p}{\partial z^{2}}=-\frac{\mu M_{0}^{2} d h / d t}{h_{0}^{2} f\left(h, l, M_{0}\right)}
$$

where, $f\left(h, l, M_{0}\right)=\frac{2 l}{A^{2}-B^{2}}\left(\frac{B^{2}}{A} \tanh \frac{A h}{2 l}-\frac{A^{2}}{B} \tanh \frac{B h}{2 l}\right)$

The roughness film thickness is consisting of two parts

$$
H(x)=h(x)+h_{s}
$$

where $h(x)$ is the mean film thickness and $h_{s}$ is a randomly varying quantity measured from the mean level and thus characterizes the surface roughness. Further, stochastic part $h_{s}$ is considered to have the probability density function $f\left(h_{s}\right)$ defined over the domain $-c \leq h_{s} \leq c$ where $c$ is the maximum deviation from the mean film thickness. The mean $\alpha^{*}$, the standard deviation $\sigma^{*}$ and the parameter $\varepsilon^{*}$, which is the measure of symmetry of the random variable $h_{s}$ are defined as:

$$
\begin{aligned}
& \alpha^{*}=E\left(h_{s}\right) \\
& \sigma^{*^{2}}=E\left[\left(h_{s}-\alpha^{*}\right)^{2}\right] \\
& \varepsilon^{*}=E\left[\left(h_{s}-\alpha^{*}\right)^{3}\right]
\end{aligned}
$$

The probability distribution function $f\left(h_{s}\right)$ for the stochastic film thickness. In many of the engineering problems, the roughness height distributions of the surfaces are found to be Gaussian in nature. A polynomial form that approximates the Gaussian is chosen such probability density function is given by

In this equation $E$ is an expectancy operator defined by

$$
E(\circ)=\int_{-\infty}^{\infty}(\circ) f\left(h_{S}\right) d h_{S}
$$

where $f\left(h_{s}\right)=\left\{\begin{array}{lc}\frac{35}{32 c^{7}}\left(c^{2}-h_{s}^{2}\right)^{3}, & -c<h_{s}<c \\ 0, & \text { elsewhere }\end{array}\right.$ and $h_{s}$ is the stochastic film thickness, $\bar{\sigma}$ is standard deviation and $c=3 \bar{\sigma}$. 
Assuming the symmetric flow of the magnetic fluid about $x=0$ between the parallel plates under an oblique magnetic field and whose magnitude is a function of $x$ vanishing at

$$
\left(x_{1}-a\right)\left(x_{1}-\sqrt{3} z_{1}+2 a\right)\left(x_{1}+\sqrt{3} z_{1}+2 a\right)=0
$$

Where, $a$ is the length of the side of the equilateral triangle whose equation is

$$
(x-a)(x-\sqrt{3} z+2 a)(x+\sqrt{3} z+2 a)=0
$$

The point of intersection of the medians of the triangle is selected as the origin

The modified stochastic Reynolds equation is found by taking the stochastic average of (9) with respect to $f\left(h_{s}\right)$

$$
\frac{\partial^{2} E(p)}{\partial x^{2}}+\frac{\partial^{2} E(p)}{\partial z^{2}}=\frac{\mu M_{0}^{2} d h / d t}{h_{0}^{2} E\left\{f\left(H, l, M_{0}\right)\right\}}
$$

where, $E\left\{f\left(H, l, M_{0}\right)\right\}=E\left\{\frac{2 l}{A^{2}-B^{2}}\left(\frac{B^{2}}{A} \tanh \frac{A H}{2 l}-\frac{A^{2}}{B} \tanh \frac{B H}{2 l}\right)+H\right\}$

Solution of equation (14) with boundary condition of (16)

$$
E(p)=-\frac{\mu M_{0}^{2} d h / d t}{h_{0}^{2} f\left(H, l, \alpha^{*}, \varepsilon^{*}, \sigma^{*}\right)} k\left(x_{1}, z_{1}\right)
$$

where $k\left(x_{1}, z_{1}\right)$ is the solution of the Poisson equationand the solution of $k\left(x_{1}, z_{1}\right)$ is by variable separable method

$$
k\left(x_{1}, z_{1}\right)=\frac{a^{2}}{3}\left(1-\frac{3}{4 a^{2}} x_{1}^{2}-\frac{3}{4 a^{2}} z_{1}^{2}-\frac{1}{4 a^{3}} x_{1}^{3}+\frac{3}{4 a^{3}} x_{1} z_{1}^{2}\right)
$$

Introducing non-dimensional quantities

$$
P^{*}=-\frac{E(p) h_{0}^{3}}{\mu(d h / d t) 3 \sqrt{3} a^{2}}, l^{*}=\frac{2 l}{h_{2}}, H^{*}=\frac{h}{h_{2}}, M_{0}=B_{0} h_{2}\left(\frac{\sigma}{\mu}\right)^{1 / 2}, \alpha=\frac{\alpha^{*}}{h_{2}}, \sigma=\frac{\sigma^{*}}{h_{2}}, \varepsilon=\frac{\varepsilon^{*}}{h_{2}^{3}}
$$

Using above values in equation (17)

$$
E(p)=-\frac{\mu M_{0}^{2} d h / d t}{h_{0}^{2} f\left(H, l, \alpha^{*}, \varepsilon^{*}, \sigma^{*}\right)} \frac{a^{2}}{3}\left(1-\frac{3}{4 a^{2}} x_{1}^{2}-\frac{3}{4 a^{2}} z_{1}^{2}-\frac{1}{4 a^{3}} x_{1}^{3}+\frac{3}{4 a^{3}} x_{1} z_{1}^{2}\right)
$$

The expression for non-dimensional pressure is of the form

$$
P^{*}=-\frac{E(p) h_{0}^{3}}{\mu(d h / d t) 3 \sqrt{3} a^{2}}=\frac{M_{0}^{2}\left(1-\frac{x}{a}\right)\left(1+\frac{\sqrt{3} z}{2 a}+\frac{x}{2 a}\right)\left(1-\frac{\sqrt{3} z}{2 a}+\frac{x}{2 a}\right)}{9 \sqrt{3} F\left(H^{*}, l^{*}, \alpha, \varepsilon, \sigma\right)}
$$

where, $F\left(H^{*}, l^{*}, \alpha, \varepsilon, \sigma\right)=\frac{l^{*}}{\left(A^{* 2}-B^{* 2}\right)}\left(g_{1}^{*}-g_{2}^{*}\right)+\left(H^{*}+\alpha\right)$ 


$$
\begin{aligned}
& g_{1}^{*}=\frac{B^{* 2}}{A^{*}}\left\{\tanh \frac{A^{*} H}{l^{*}}+\frac{A^{*}}{3 l^{* 3}}\left(1-\tanh ^{2} \frac{A^{*} H}{l^{*}}\right)\left(3 l^{* 2} \alpha-A^{* 2} \varepsilon-A^{* 2} \alpha^{3}-3 A^{* 2} \sigma^{2} \alpha\right)\right\} \\
& g_{2}^{*}=\frac{A^{* 2}}{B^{*}}\left\{\tanh \frac{B^{*} H^{*}}{l^{*}}+\frac{B^{*}}{3 l^{* 3}}\left(1-\tanh ^{2} \frac{B^{*} H^{*}}{l^{*}}\right)\left(3 l^{* 2} \alpha-B^{* 2} \varepsilon-B^{* 2} \alpha^{3}-3 B^{* 2} \sigma^{2} \alpha\right)\right\}
\end{aligned}
$$

Let the equation for load carrying capacity is

$$
\begin{aligned}
& E(W)=\int_{-2 a}^{a} \int_{-\frac{2 a+x}{\sqrt{3}}}^{\frac{2 a+x}{\sqrt{3}}} E(p) d z d x \\
& E(W)=-\left(\frac{27 \sqrt{3} a^{4}}{60}\right) \frac{\mu M_{0}^{2} d h / d t}{h_{0}^{2} f\left(H, l, \alpha^{*}, \varepsilon^{*}, \sigma^{*}\right)}
\end{aligned}
$$

Thus, the equation for load carrying capacity in non-dimensional form is

$$
W^{*}=-\frac{E(W) h_{0}^{3}}{27 \mu(d h / d t) a^{4}}=\frac{\sqrt{3} M_{0}^{2}}{60 F\left(H^{*}, l^{*}, \alpha, \varepsilon, \sigma\right)}
$$

The expression for time height relationship is

$$
t=-\frac{27 \sqrt{3} a^{4}}{60 E(W)} \int_{h_{0}}^{h_{1}} \frac{d h}{\left\{\frac{h_{0}^{2}}{\mu M_{0}^{2}} f\left(H, l, \alpha^{*}, \varepsilon^{*}, \sigma^{*}\right)\right\}}
$$

The equation for time height relationship in non-dimensional form is

$$
T^{*}=\int_{1}^{t_{1} / t_{0}} \frac{E(W) h_{0}^{2} d t}{27 \mu a^{4}}=-\frac{\sqrt{3} M_{0}^{2}}{60} \int_{1}^{h_{1}^{*}} \frac{d h^{*}}{F\left(H^{*}, l^{*}, \alpha, \mathcal{\varepsilon}, \sigma\right)}
$$

\section{RESULTS AND DISCUSSIONS}

The influence of magnetic field on squeeze film lubrication of triangular plates is discussed. The squeeze film characteristics are obtained as the function of the Variance $\alpha$ and Hartmann number $M_{0}$ signifies the effect of magnetic field. Hence in the present analysis the following range of values of non-dimensional parameters are used: variance $(\alpha=-0.1-0.1)$, couple stress parameter $\left(l^{*}=0-0.4\right)$, Measure of symmetry parameter $(\varepsilon=-0.1-0.1)$ and Hartmann number $\left(M_{0}=0-4\right)$.

\subsection{Non-Dimensional Pressure}

Figure 2 Depicts the variation of non-dimensional pressure $P^{*}$ with $x^{*}$ for different values of couple stress parameter $l^{*}$ and Hartmann number $M_{0}$ with $h^{*}=1.2, y^{*}=0, \alpha=0.005, \varepsilon=0.05, \sigma=0.1$. It is observed that the non-dimensional pressure increases with increasing the values of couple stress parameter and Hartmann number $M_{0}$ as compared to the Newtonian case $\left(l^{*}=0\right)$ and non-magnetic case $\left(M_{0}=0\right)$. Further it is observed that the non-dimensional pressure is more 
significant in non-Newtonian case. Figure 3 Shows the variation of non-dimensional pressure $P^{*}$ as a function of $x^{*}$ for different values ofvariance $\alpha$ with $M_{0}=0.2, l=0.4, h^{*}=1.2, y^{*}=0, \varepsilon=0.05, \sigma=0.1$. It is observed that, pressure $P^{*}$ decreases for negatively skewed $\alpha$.Variation of non-dimensional pressure $P^{*}$ with $x^{*}$ for different values $\varepsilon$ with $h^{*}=$ $1.2, y^{*}=0, M=2, l=0.4, \alpha=0.005, \sigma=0.1$,is displayed in Figure 4 . It is found that the non-dimensional pressure $P^{*}$ decreases for increase of $\varepsilon$. Figure 5 shows the variation of non-dimensional pressure $P^{*}$ as a function of $x^{*}$ for various values of standard deviation $\sigma$ with $M_{0}=2, l^{*}=0.4, h^{*}=1.2, y^{*}=0, \varepsilon=0.05$. it shows that the non-dimensional pressure decreases for increasing the values of $\sigma$.
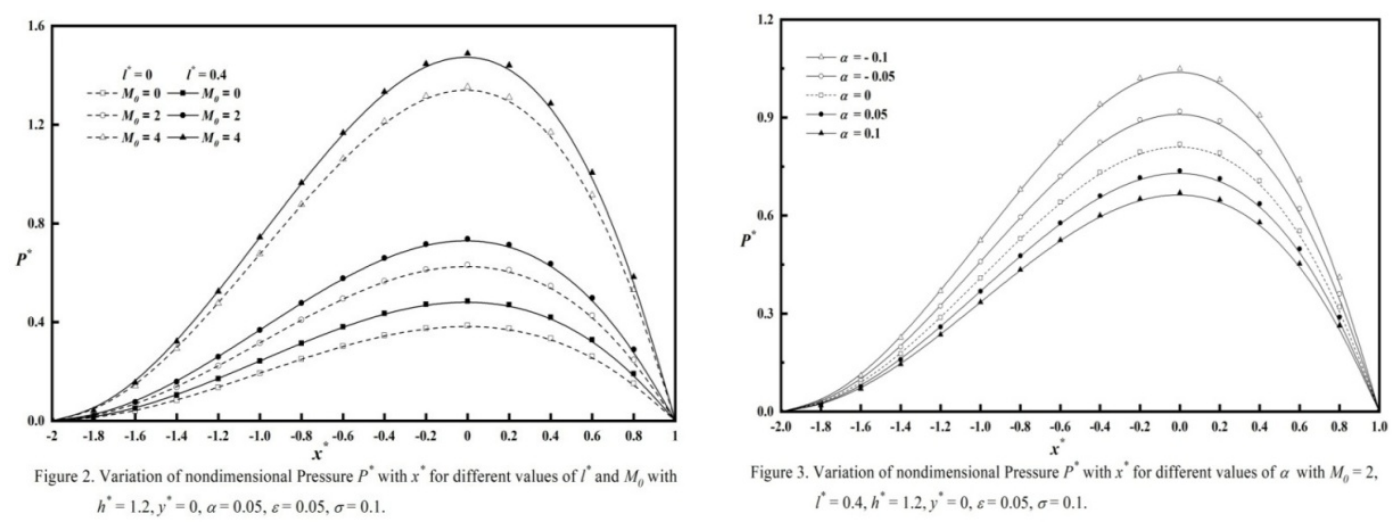

Figure 2
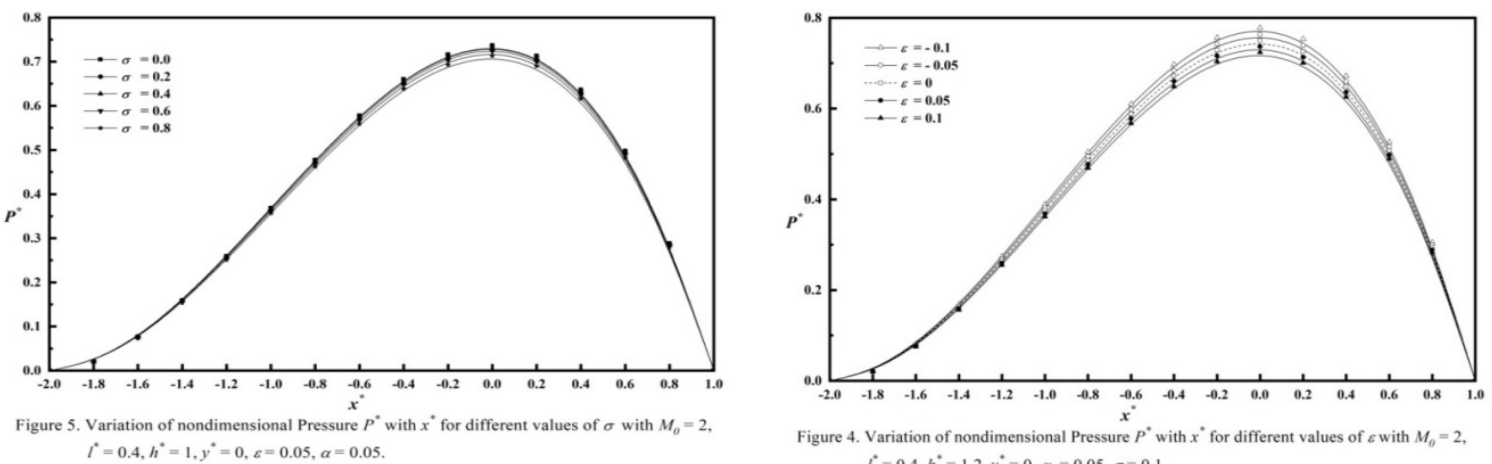

$i^{\circ}=0.4, h^{\circ}=1.2, y^{\circ}=0, \alpha=0.05, \sigma=0.1$

Figure 3
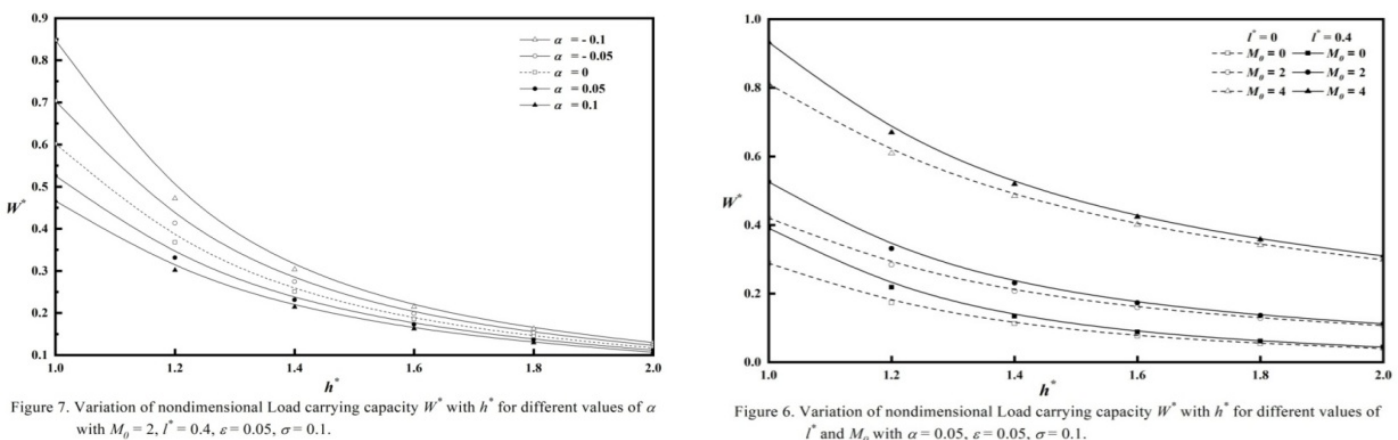

Figure 4

\subsection{Non-Dimensional Load Carrying Capacity}

Figure 6 Represents the variation of non-dimensional load carrying capacity $W^{*}$ with film thickness $h^{*}$ for different values 
of couple stress parameter $l^{*}$ and Hartmann number $M_{0}$ with fixed values of $\alpha=0.05, \varepsilon=0.05, \sigma=0.1$. It is found that the non-dimensional load carrying capacity increases for increasing values of transverse applied magnetic field $M_{0}$. Further it is noticed that the load carrying capacity is more significant as compared to the non-Newtonian case. Figure 7 depicts the variation of load carrying $W^{*}$ with $h^{*}$ for various values of $\alpha$ with fixed parameter $M_{0}=2, l^{*}=0.4$, $\varepsilon=0.05, \sigma=0.1$. It clear that the load carrying capacity decreases for increasing values of $\alpha$. Variation of load carrying capacity $W^{*}$ with film thickness $h^{*}$ for different values of measure of symmetry $\varepsilon$ is shown in Figure 8 It shown that the load carrying capacity decreases as increasing the smaller values $\varepsilon$. Figure 9 depicts the variation of load carrying capacity versus film thickness for different values of $\sigma$. Load carrying capacity decreases with increase of standard deviation $\sigma$.

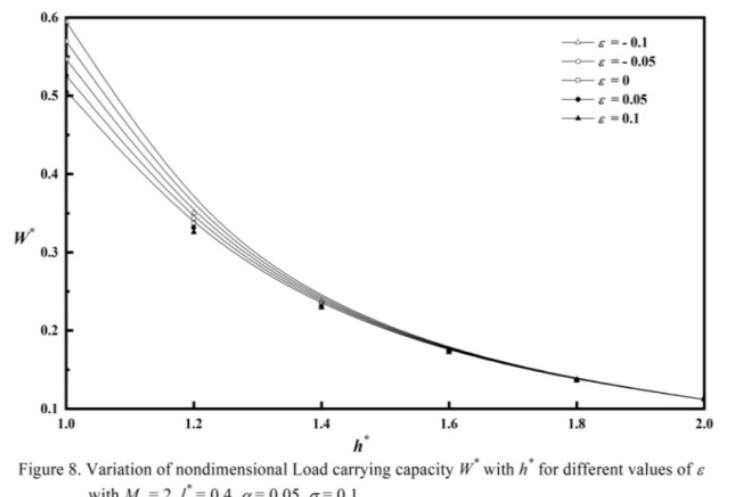

with $M_{0}=2, l^{\circ}=0.4, \alpha=0.05, \sigma=0.1$.

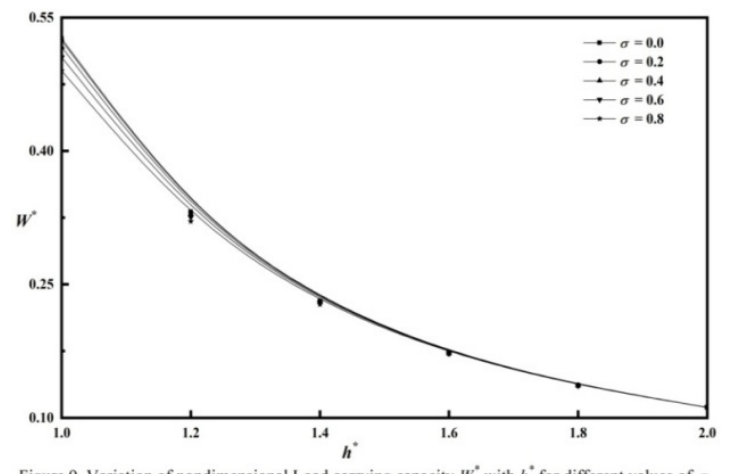

Figure 9. Variation of nondimensional Load carrying capacity $W^{*}$ with $h^{*}$ for different values of $\sigma$ with $M_{0}=2, l^{\circ}=0.4, \varepsilon=0.05, \alpha=0.05$.

Figure 5

\subsection{Non-Dimensional Squeeze Film Time}

Variation of non-dimensional squeeze film $T^{*}$ versus $h_{1}^{*}$ for different values of couple stress parameter $l^{*}$ and Hartmann number $M_{0}$ for fixed values of $\alpha=0.05, \varepsilon=0.05, \sigma=0.1$. is depicted in Figure 10. It found that increase in squeeze film time as increase of applied magnetic field and couple stress parameter. Further it is to be found that the non-dimensional squeeze film time $T^{*}$ is more significant in Newtonian case. Figure 11 and Figure 12 shows the variation of nondimensional squeeze film time $T^{*}$ versus film thickness $h_{1}^{*}$. It is found that the decrease of squeeze film time in increase smaller values of $\alpha$ and $\varepsilon$. Figure 13 represents the variation of squeeze film time $T^{*}$ versus film thickness $h_{1}^{*}$ for various values $\sigma$ for fixed $M_{0}=2, l^{*}=0.4, \varepsilon=0.05$, and $\alpha=0.05$. It is observed that squeeze film time gradually decreases due to increase in $\sigma$.

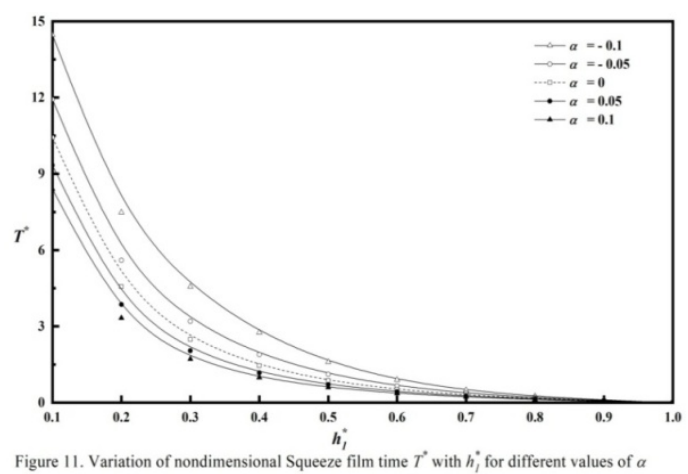

with $M_{\theta}=2, l^{\circ}=0.4, \varepsilon=0.05, \sigma=0.1$.

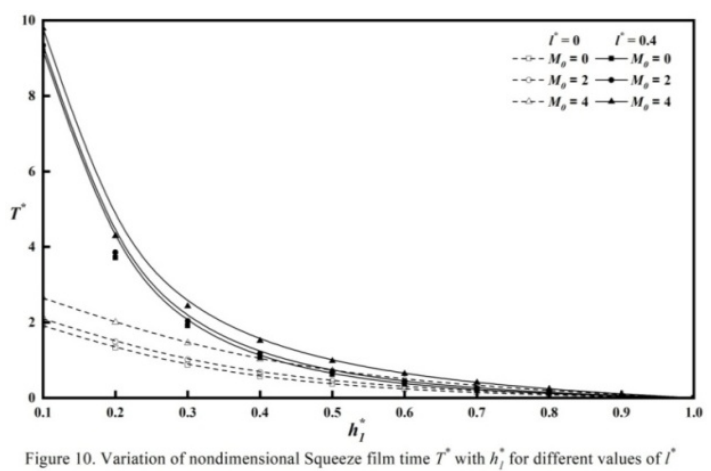

and $M_{0}$ with $\alpha=0.05, \varepsilon=0.05, \sigma=0.1$.

Figure 6 

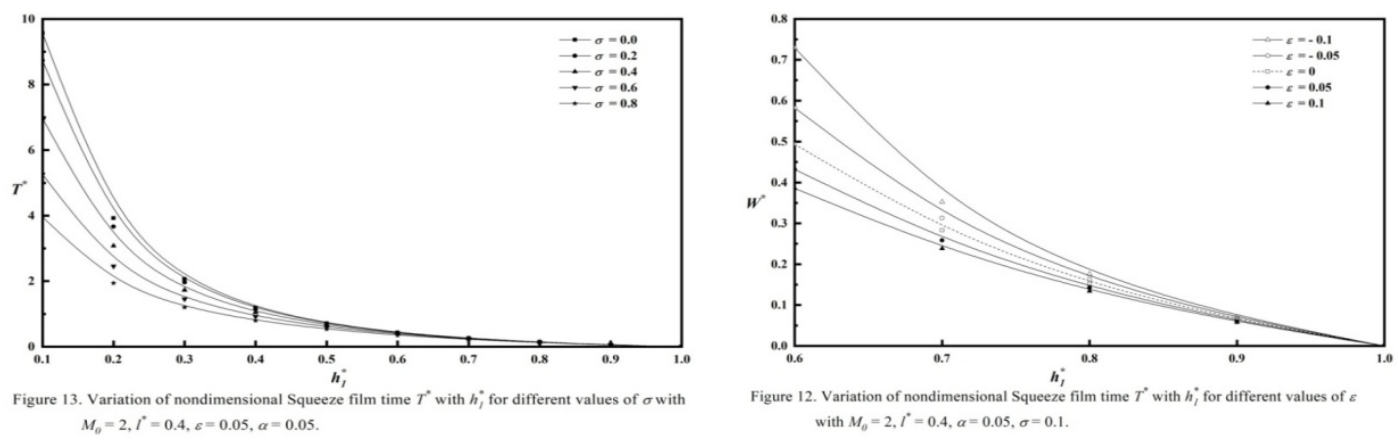

Figure 7

\section{CONCLUSIONS}

It is acknowledged from this study that the conflicting effect of the standard deviation and measure of symmetry can be minimized by the magnetic field and couple stress lubricant in the case of negatively skewed roughness when negative variance occurs. Further, this analysis makes amendatory that the roughness given expected consideration while designing the bearing system even if the relevant values of Hartmann number and couple stress lubricant.

\section{REFERENCES}

1. Huges W F. and Elco R A., Magneto hydrodynamics flow between parallel rotating dicks. J.Fluid Mech., Vol.13 (1).

2. Snyder, W T., “The magneto hydrodynamics slider bearing," Journal of Basic Engineering, Vol. 84(1962), 197204.

3. Hughes W F., "The Magneto hydrodynamics lubrication of finite slider bearing”, Journal of Basic, Engineering, Vol.85,(1963) 12936.

4. Davies, M G., The generation of pressure between rough fluid lubricated moving deformable surfaces. Lubrication Engineering, Vol. 102(1963) 4850.

5. Dudzinsky $S$ J., Young F J. and Huges WF., on the load capacity of the MHD journal bearing, Journal of Lubrication Technology, Vol.90 (1) (1968) 139144.

6. Stokes, V K. Couple stresses in fluids. The Physics of fluids, Vol.9 (9):1966. (1709)- (1715).

7. Bujurke, N M and jayaramanG. The influence of couple stresses in squeeze films. International Journal of Mechanical Sciences, Vol.24 (6) (1982)369376.

8. $\quad$ Ramaniah, G. Squeeze films between finite plates lubricated by fluids with couple stress. Wear, Vol.54 (2) (1979) 315320.

9. Lin, J R.,Squeeze film characteristics between a sphere and a flat plate couple stress fluid model. Computers and structures, Vol.75 (1) (2000):7380.

10. Lin JR., Magneto- hydrodynamics squeeze film characteristics for finite rectangular plates, Industrial lubrication and Technology, Vol.55 (2) (2003), 8489.

11. Naduvinamani N B and Siddangouda A. squeeze film lubrication between circular stepped plates of couple stress fluids, $J$ of the Braz.Soc. of Mech, Vol.31(1) (2009) 16.

12. Naduvinamani N B., Fathima S T. and Hanumagouda B N., Magneto- hydrodynamic couple stress squeeze film lubrication of circular stepped plates, Journal of Engineering Tribology 2010:225 (Part-I): 1119.

13. Naduinamani, N B. and Rajashekar M.. MHD and couple stresses squeeze film characteristics between sphere and plane 
surface, Tribology Material surface and interfaces, Vol.5 (3) (2011)9499.

14. Turaga.R., Sekhar A S. and Majumdar B C., The effect of roughness parameter of hydrodynamic journal bearings, Tribol Trans. Vol.44(2), 291297.

15. Naduvinamani N B. and Gurubasavaraj G., Surface Roughness Effects on squeeze films in Curved Circular Plates, Industrial Lubrication Tribology, Vol.56(6), (2004) 346352.

16. Rajashekar M and Biradar K. Effect of surface roughness on MHD couple stress squeeze film characteristics between a sphere and porous plane surface Advance in Tribology, 2012, 111.

17. Basti,D P., Effect of surface roughness and couple stress on squeeze films betweencurved annular plates. ISRN Tribology, 2013: 18.

18. Fathima, S T. Naduvinamani N B. Hanumagouda B N and Santosh kumar J. Modified Reynolds equation for different type of finite plates with combined effects of MHD and couple stress, Tribology Transaction, Vol.58(4),(2014) 660667.

19. Naduvinamani N B., Hiremath P S. and Gurubasavaraj G. Effect of surface roughness on the couple stress squeeze film between a sphere and a flat plate. Tribology International, Vol.38 (5) (2005) 451458.

20. Naduvinamani N B., Siddangouda A. and Siddharam P., Effect of surface roughness on static and dynamic characteristic of MHD couple stress lubrication of parabolic slider bearing. Tribology Material Surfaces and Interfaces, Vol.11 (20) (2017)107115.

21. Aryawan, W. D., \& Putranto, T. The Hydrodynamics Performance Of Aquaculture Fishing Vessel In Variation Of Deadrise Angle And Sponson.

22. Anand, A., Krishna, K. H., Ammineedu, E., \& Rao, B. S. P. Temporal Variation Of Ground Water Quality In Central Godavari Delta And Its Significance In Hydrodynamics Of The Area.

23. Bahuguna, S. (2015). Simulation of Particle \& Liqiud Impact on Water Film Under Equidense Conditions using Smoothed Particle Hydrodynamics (SPH). International Journal of Mechanical and Production Engineering Research and Development (IJMPERD), 5(6), 18.

24. Ghosh, K., Kumar, G. K., \& Pai, C. D. Y. (2018). Free Vibrational Analysis Ofmagneto-Rheological Aircraft Rib Structure. 